at the end of a main meal, and preferably only once a day. It is the almost continuous exposure of the plaque on teeth to fermentable carbohydrate that leads to rampant caries. Sweeteners on dummies and minifeeders containing sweetened solutions can devastate a small child's dentition. The gifts of sweet-giving friends and sugary "pop" are as dangerous for older children. Regrettably, many drugs are made up in paediatric mixtures containing high concentrations of sugar. This may not matter for the child who needs an occasional course of treatment, but it can be harmful for children on regular medication.

General practitioners may be asked about the value and safety of water fluoridation. Neither is in doubt. The toxicology of fluoride is extensively documented and few other subjects have been so fully investigated. Fluoridation of water is by far the simplest and safest way of providing the necessary small doses of fluoride, particularly for children. Incorporation of the right amount of fluoride in the teeth does not protect against the effects of excessive sugar, but it will reduce the number of cavities at an age when their repair is not easy. To maintain the beneficial effect through adult life the concentration of fluoride in the surface enamel must be maintained. Fluoride toothpaste used regularly will do this. Plain toothpaste should be changed to fluoride toothpaste as soon as the child can rinse and spit without swallowing.

Finally, doctors can help to save fractured teeth. A fractured or subluxated tooth is an emergency. If treated within hours most can be saved. Neglected for even a day or two, many will be lost.

${ }^{1}$ British Paediatric Association and British Paedontic Society, The Dental Health of Children. London, British Paediatric Association, 1979.

\section{Diagnosis of cholestasis}

Some spectacular progress has been made in gastroenterology as a result of the invention and development of fibreoptic endoscopy, ultrasonography, and, more recently, computerised tomography. One topic of change is the diagnosis of cholestatic jaundice. Though its cause can often be diagnosed on the basis of a careful history and examination, the differing managements of intrahepatic and extrahepatic cholestasis make early proof of diagnosis essential. Conventional blood tests will usually confirm the presence of cholestasis, but they provide little and often misleading information about its cause. Newer tests such as estimating the serum concentrations of bile acids and lipoprotein $\mathrm{X}$ have proved equally disappointing.

Various procedures have been devised to help in diagnosis, including percutaneous transhepatic cholangiography (PTC), endoscopic retrograde cholangiopancreatography (ERCP), and grey-scale ultrasonography. Of these, ultrasound scores high on safety and simplicity, and its relative cheapness adds to its attractions. Nevertheless, it is a new procedure and still under evaluation. In one series of 26 cases of cholestasis studied consecutively" extrahepatic "surgical" obstruction was diagnosed ultrasonically in nine out of 10 cases, and intrahepatic "medical" causes were found in all 14 others (with two failures of examination). This report laid particular emphasis on the importance of gall-bladder dilatation; absence of this sign was misleading in only one patient. Stones in the gall bladder were an incidental finding in several cases.
In another small series ${ }^{2}$ of grey-scale ultrasonograms in cholestasis, dilated ducts were found in eight out of 13 cases with extrahepatic obstruction, and there were no false-positive reports of dilatation of the ducts in seven cases with intrahepatic cholestasis. A larger and more fully documented study $^{3}$ of 143 jaundiced patients examined consecutively found that the size of the bile ducts could be estimated in $74 \%$ of 82 surgical and $38 \%$ of 61 medical cases. Nevertheless, $10 \%$ of the results were non-conclusive, often because of earlier biliary surgery. More disturbing was the finding that in $10 \%$ of patients with surgically remediable obstruction (mostly sclerosing cholangitis) there was no dilatation of the bile ducts. Nevertheless, in $22 \%$ of cases grey-scale ultrasonography was the only diagnostic procedure considered 'necessary.

A more recent series of 55 consecutive patients yielded some apparently better results. ${ }^{4}$ There was only one technical failure, and the calibre of the bile ducts was incorrectly reported in a further case. All 14 cases with intrahepatic cholestasis were identified correctly, with complete diagnosis in four patients with cirrhosis and one with liver metastases. There were 41 patients with extrahepatic obstruction and a complete diagnosis was made in 23 of these. By no means all of these patients had dilatation of the biliary tract: only five out of 14 patients considered to have obstructive jaundice secondary to gall stones had dilated bile ducts, and in five others the presence of stones in the gall bladder alone was taken as indicating the diagnosis. This is a crucial point, since the authors recommend surgical procedures without recourse to PTC or ERCP for such patients-a policy that cannot be condoned, because not only are gall stones very common, but their frequency is doubled in cirrhosis, ${ }^{5}$ and they will often be entirely incidental to the cause of jaundice.

If dilated ducts are detected then PTC should confirm extrahepatic obstruction in $90-100 \%$ of patients. ${ }^{6}$ Failure with this procedure strongly suggests a non-surgical lesion. Nevertheless, even the fine Chiba needle technique carries appreciable complication $(2 \cdot 6-5 \cdot 0 \%)$ and mortality rates $(0.25 \%)$; these rates are similar to those with the older sheathed needle. Lack of urgency in arranging surgery may be one factor in this morbidity and mortality. In expert hands ERCP will provide a diagnosis of the presence of cholestatic jaundice in $90 \%$ of cases $^{7}$; but the technique is difficult to master and the failure rate is $13-62 \%$, with a complication rate of $2-15 \%$ and a mortality rate of $0 \cdot 1-2 \%{ }^{78}$

If there is no evidence of duct dilatation or choledocholithiasis, and blood coagulation is normal, then liver biopsy is appropriate and safe to establish the diagnosis. In cases of doubt a repeat ultrasonogram carries no hazard apart from possible delays.

With increasing skill ultrasonography may eventually become the crucial investigation in patients with suspected extrahepatic obstruction, giving complete diagnosis without the need for invasive tests. At present it should be regarded as a preliminary screening test before cholangiography or biopsy. ${ }^{9}$

1 Vicary, F R, et al, Gut, 1977, 18, 161.

${ }^{2}$ Morris, A H, et al, Gut, 1978, 19, 685.

3 Sample, W F, et al, Radiology, 1978, 128, 719.

4 Vallon, A G, Lees, W R, and Cotton, P B, Gut, 1979, 20, 51.

${ }^{5}$ Bouchier, I A D, Gut, 1969, 10, 705.

${ }^{6}$ Elias, E, Gut, 1976, 17, 801.

7 Cotton, P B, Gut, 1977, 18, 316.

${ }^{8}$ Bilbao, M K, et al, Gastroenterology, 1976, 70, 314.

9 Taylor, K J W, and Resenfield, A T, Clinics in Gastroenterology, 1978, 7, 488. 